\title{
Evaluation of the Mini Nutritional Assessment short-form among the elderly population from Ethiopia
}

Megersso Urgessa ( $\square$ megurgessa@gmail.com )

Madda Walabu University Shashemenne campus

\section{Research Article}

Keywords: MNA-SF, Meki, geriatrics, Ethiopia

Posted Date: July 13th, 2021

DOI: https://doi.org/10.21203/rs.3.rs-637038/v2

License: (c) (i) This work is licensed under a Creative Commons Attribution 4.0 International License.

Read Full License 


\section{Abstract}

Background Different tools have been used to perform a nutritional screening and assessment, and MNA is one of the widely used and recommended tools in the geriatrics population. MNA has two forms, long and short. However, MNA short-forms have not been evaluated in Ethiopia. Therefore, this study was aimed to evaluate MNA short form against MNA long-form tool among Ethiopian elders.

Methods One hundred and seventy-six randomly selected elders entered into the community-based crosssectional validation study. Amputated, bedridden, those with visible deformity were excluded. Original MNA questionnaires were translated to Afan Oromo and Amharic languages. All translated and pretested MNA questionnaires were administered to each participant. The anthropometrics were measured. Reliability, validity, sensitivity, specificity, positive and negative predictive values were calculated. Receiver-operating characteristic curve (ROC-curve) analysis was plotted for MNA, to identify the area under the curve (AUC) and optimal cut-off value for prediction of malnutrition.

Result Strong association between MNA-long form score and MNA-short form score indicated by spearman's rank correlation coefficients of BMI-MNA-SF 0.771, $\mathrm{p}<0.05$ and CC-MNA-SF 0.759, $\mathrm{P}<0.05$. Similar the agreement between the long and short form of MNA was found to be a weighted kappa $0.396(0.318,0.474)$ for BMI-MNA-SF and $0.546(0.422,0.669)$ for CC-MNA-SF at $95 \% \mathrm{Cl}$. These values indicate moderate agreement with the MNA-long form. There is very good agreement between the BMIMNA-SF and CC-MNA-SF $0.400(0.322,0.478)$. Also, high power to identify two categories using MNA long-form as golden standard with AUC for BMI - MNA-SF 0.908 (0.865-0.951) and 0.880 (0.831-0.929) for CC-MNA-SF at $95 \% \mathrm{Cl}$. Diagnostic accuracy of both versions of MNA-SF showed that $34.2 \%$ sensitivity, $100.0 \%$ specificity, $100.0 \%$ PPV, and $41.5 \%$ NPV for BMI-MNA-SF. Similar sensitivity $75.8 \%$, specificity 83.9\%, PPV 91.0\%, and 61.8\% NPV for CC-MNA-SF. Total Diagnostic accuracy for BMI-MNA-SF 55.12\%, and $78.41 \%$ for CC-MNA-SF

Conclusion Both versions of MNA-SF were found to be valid screening tools in the Ethiopian elders against Long-form MNA.

\section{Introduction}

Elderly people refer to older persons aged 60 and above years $(1,2)$. In this 21 st century, the world population is radically increasing aging at the fastest rate. By 2050, elderly population aged 60 and above will double from 12-22\%. Particularly will increase in developing country(3). In one of the developing countries found in East Africa, Ethiopia, also this age group is dramatically increasing with aged 60 years and above is more than three million with $4.42 \%$ of the total population are living in Urban(1). Moreover, the country's life year expectancy is raised to be 67.8 years $(4,5)$. Because of various factors especially those related to aging and physiologic change in this age group, the elderly peoples are highly vulnerable to several degenerative diseases and malnutrition (6). Due to these currently, this age group is affected by the dual-burden of chronic non-communicable diseases and malnutrition $(7,8)$. 
Malnutrition is defined as over or under consumption of nutrients, those very crucial for the health and growth of elderly people (9). However, here malnutrition was used to refer to undernutrition.

Undernutrition, a condition resulting from inadequate consumption of nutrients(10), is a specific concern in the elderly population because it leads to different complications including morbidity and mortality(11). This type of malnutrition is almost undiagnosed and its magnitude varies from setting to setting. In developed countries, the incidence found that $15 \%$ in the community, $23-62 \%$ in hospitals, and more than $80 \%$ in care units (12). In developing countries similarly incidence varies from country to country, for instance, in South Africa $50 \%$ in the hospital(13), in Chile $58 \%$ in hospital (6), Egypt $26.5 \%$ in the community (14), and Ethiopia $28.3 \%$ were malnourished in the community (15). Given that the elderly population is increasing, the prevalence of undernutrition among the elderly population will also increase. Therefore, it is crucial to arrange programs at all levels that enable the early detection of at risk for malnutrition, and that followed by appropriate intervention. Moreover, it has been proposed that early detection using valid malnutrition screening tool is help to prevent malnutrition and its complication (16).

Malnutrition screening is a rapid and easy process using a valid malnutrition screening tool, aimed to detect elderly people who may need intervention(17). Malnutrition screening tools are mostly structured questionnaires, containing risk factors for malnutrition (for instance, difficulty of chewing, appetite loss, or functional limitations) and indicators of malnutrition(for instance, involuntary recent weight loss) (18). Moreover, they are simple and administered by any trained professional. However, the validity of these tools is very crucial to carry out any screening process.

A valid tool is a tool that measures what it is suggested to measure. Valid tools ensure the accurate detection of those at risk for malnutrition and facilitate nutritional intervention (19). Validity of tool usually measured by correlation with a golden standard tool (criterion-related validity) (20). There are different valid screening tools used in the geriatric field to screen malnutrition, among these MNA is a widely used and valid malnutrition screening tool for different country's elders (21).

MNA was developed in the early 1990s and published in 1994 (22). It has two forms, short and long. Both types of MNA can be used in the community and health care setting $(23,24)$. The MNA long-form has 18 items with a maximum of 30 points, completed in 10-15 minutes (22). Subsequently, Rubenstein and colleagues developed a short form that only contains six items from eighteen that complete within 3 to 5 minutes to overcome the time burden of MNA long-form. Also, this tool has two forms body mass index (BMI)- MNA short-form and calf circumference (CC)-MNA short-form (25). The first main aim of this short form is to categorize the geriatric population's nutritional status as well-nourished or at risk for malnutrition, and then the professional needs only the MNA long-form if the subjects were categorized as at risk for malnourishment. But, currently works alone to categorize into three categories including malnutrition (24).

Moreover, the practical advantage of short-form was tested by multiple screening instruments such as the malnutrition universal screening tool(26), short nutritional assessment questionnaire(27), and nutritional risk screening 2002(18). This mini nutritional assessment was used BMI mostly even though some Asian 
and Africa population weight was not a common health measure, instead, they use CC and mid-upper arm circumference(MUAC) with exception nutrition screening tool for South African elder include only mid-upper arm circumference(28). However, MNA short form uses both BMI and CC. In addition, ten years ago MNA short-form tool was validated and at the time it has high sensitivity, high specificity, and high correlation with long-form MNA (29). The first main aim of this short form is to categorize the geriatric population's nutritional status as well-nourished or at risk of malnourishment, and then the professional needs only the full form if the subjects are categorized as at risk for malnourishment. But, it works alone to categorize into three categories including malnutrition (24). Still, nowadays MNA short-form is a valid and recommended tool used to assess nutritional status among elderly people in a different country (30).

Even though this tool is validated and used in a different country, it is not readily applicable to other countries. This because population characteristics are varying from country to country especially in terms of anthropometric measurement and nutritional characteristics. However, only long-form MNA was validated for Ethiopian elders $(15,31)$. MNA short-forms have not been validated for the Ethiopian elderly and there is a research gap on whether the MNA short-forms and its established cut-off point are applicable to screen and assess malnutrition among the elderly population in the Ethiopian context. Therefore, this study was done to validate MNA short-forms using MNA long-forms as a golden standard in Ethiopia.

\section{Methods And Materials}

\section{Participants}

The study was conducted in Meki town, East Ethiopia, in 2020. One hundred and seventy-six elders were entered into this study randomly using a sample frame developed after house-to-house elderly people surveyed. All elderly people aged 60 and above years were included. Whereas an elderly person, who was amputated, bedridden and has a visible deformity were excluded.

\section{Nutritional assessment}

MNA long-form is used to identify malnutrition, at risk for malnutrition, and well-nourished elderly people. It classifies as malnutrition less than 17 points, at risk of malnutrition 17 to 23.5 points, and wellnourished: 24 to 30 points $(32,33)$

Novel MNA questionnaires were translated to local language and administered to all participants after doing the pretest. All participant's weight, height, MUAC and CC were measured. Each measured twice and the average record was used for this study. Height was measured using a stadiometer (Seca 213, Germany), with participant bare feet, buttock, heels, and occiput part touch board. The participant's height was recorded to the nearest 0.1 centimeters $(\mathrm{cm})$. The weight was measured using calibrated digital scales placed on a hard flat surface with the participant in light clothes, bare feet, and recorded to the nearest 0.1 kilograms $(\mathrm{kg})$. The weighing scale was checked after each measurement with a $2 \mathrm{~kg}$ standard weight. MUAC was measured at the mid-point between the tip of the Acromion and Olecranon 
process on the back of the upper arm while the subject's forearm held a freely horizontal position and recorded nearest $0.1 \mathrm{~cm}$. CC was measured at the widest circumference between ankle and knee to the nearest $0.1 \mathrm{~cm}$ using flexible tape in a sitting position with leg 900 at the knee. Body mass index (BMI) computed as body weight in kilograms divides squares of height in meters. All data were collected by trained BSc Nurses and BSc Public health.

Data processing and analysis

The data were entered into Epidata version 3.1, then exported and analyzed by SPSS version 25. The variables of interest were described by using means, standard deviations. A P-value $<0.05$ was used to define statistical significance.

To evaluate the reliability, the overall internal consistency of the MNA short forms were evaluated by Cronbach's alpha. The alpha value of $0.60-0.70$ acceptable, $0.70-80$ adequate, and $\geq 80 \operatorname{good}(34)$. Also, MNA short forms correlation with its 6-item assessed by Spearman's rank correlation coefficient.

Criterion-related validity MNA short-forms were evaluated by Spearman's rank correlation coefficient. Spearman's rank correlation coefficient value: 0.90 - 1.00 very high, $0.70-0.90$ high, $0.50-0.70$ moderate, $\leq 0.50$ lower (35).

The inter-method agreement was assessed by weighted kappa, between the MNA short-form and MNA long-form, using $3 \times 3$ cross-tabulation. Weighted kappa value:0.80-1.0 perfect agree,0.61-0.80 substantial, $0.41-0.60$ moderate, $0.21-0.40$ fairly (36).

Sensitivity, specificity, PPV, and NPV of MNA short-forms were calculated by $2 \times 2$ cross-tabulation using MNA long forms a golden standard. Markers of malnutrition MNA long-form score $<24$ points or MNA short-forms $<11$ points.

To determine AUC and new optimal cut-off value, the MNA short-form tool's ROC curve was plotted using MNA long-form $<24$ points as markers of malnutrition. The AUC was used to assess the overall accuracy of the MNA tool. AUC value $\geq 0.9$ excellent,0.8-0.9 Good, 0.7-0.8 satisfactory and 0.6-0.7 not good (37). A new optimal cutoff values were calculated using Youden's index (sensitivity + specificity -1$)(38)$.

\section{Results}

Characteristics of study participants

Total one hundred and seventy-six elders participated in the study. From this, 78(44.3\%) were males. The mean (SD) age of the participants was $67.56( \pm 5.791)$ years and ranged from 60 to 84 years. Overall, the mean (SD), total MNA score, BMI-MNA-SF and CC-MNA-SF of the participants were $20.70 \pm 3.46,11.78 \pm$ 1.74 and $9.99 \pm 1.64$ respectively (Table 1 ). 
Table 1

Characteristics of study participants elderly people aged 60 and above years in the community, Meki town, East Ethiopia, 2020.

\begin{tabular}{|lll|}
\hline Sex & Male (no, \%) & $\mathbf{7 8 ~ ( 4 4 . 3 \% ) ~}$ \\
\cline { 2 - 3 } & Female (no, \%) & $\mathbf{9 8 ~ ( 5 5 . 7 \% ) ~}$ \\
\hline Age category in year) (no, \%) & $60-64$ & $61(34.7 \%)$ \\
\cline { 2 - 3 } & $65-69$ & $63(35.8 \%)$ \\
\cline { 2 - 3 } & $70-74$ & $24(13.6 \%)$ \\
\cline { 2 - 3 } & $75-79$ & $23(13.1 \%)$ \\
\hline Age in year (mean, SD) & & $5(2.8 \%)$ \\
\hline Weight in Kg (mean, SD) & & $67.56(5.79)$ \\
\hline Height in meters (mean, $S D)$ & & $70.72(10.15)$ \\
\hline MNA (sum score) (mean, $S D)$ & & $1.70(0.07)$ \\
BMI-MNA- short form(sum score) (mean, SD) & $11.78(1.74)$ \\
CC-MNA-short form(sum score) (mean, $S D)$ & $9.99(1.67)$ \\
\hline
\end{tabular}

Reliability of MNA

The overall homogeneity between the six MNA-SF items was adequate with Cronbach's Alpha of 0.205 for BMI-MNA-SF and 0.319 for CC-MNA-SF (Table 2). In addition, both version MNA-SF total score significantly correlate with all their items at Spearman's rho $>0.759$, P-value $<0.05$. 
Table 2

Cronbach's alpha for the MNA-SF tool applied in the elderly population aged 60 and above years in the community, Meki town, East Ethiopia, 2020.

\begin{tabular}{|lll|}
\hline Items & $\begin{array}{l}\text { Cronbach's a } \\
\text { (BMI-MNA-SF) }\end{array}$ & $\begin{array}{l}\text { Cronbach's a } \\
\text { (CC-MNA-SF) }\end{array}$ \\
\hline 1. Decreased food intake & .065 & .234 \\
\hline 2. Weight loss & .085 & .0249 \\
\hline 3. Mobility status & .219 & .333 \\
\hline 4. Acute stress & .171 & .246 \\
\hline 5. Depression & .213 & .310 \\
\hline 6. BMI/CC category & .277 & .277 \\
\hline Overall Cronbach's alpha & 0.205 & 0.319 \\
\hline
\end{tabular}

Validity of MNA

Criterion-related validity of the BMI-MNA-SF and CC-MNA-SF tools were significant as compared to MNA long form with correlation coefficient spearman's rho (rs) of 0.771 and 0.759 respectively (Table 3). According original cut off point BMI-MNA-SF had sensitivity $34.2 \%$, specificity of $100 \%$, PPV of $100 \%$, and NPV $41.5 \%$ of MNA with a total diagnostic accuracy of $55.12 \%$ (Table 3 ). Similarly for CC-MNA-SF had sensitivity $75.8 \%$, specificity $83.9 \%$ PPV $91.0 \%$ and NPV $61.8 \%$ of MNA with a total diagnostic accuracy of $78.41 \%$ 
Table 3

Measure of correlation, agreement, and diagnostic test between MNA-SF and MNA-LF of participant elderly aged 60 and above years in the community, Meki town, East Ethiopia, 2020.

\section{MNA-SF correlation by Spearman's rho(rs)}

\begin{tabular}{lll|}
\hline BMI-MNA-SF With MNA-LF & 0.771, P-value $<0.05$ & \\
\hline CC-MNA-SF With MNA-LF & 0.759, P-value $<0.05$ & \\
\hline MNA-SF agreement with MNA-LF & BMI-MNA-SF & CC-MNA-SF \\
\hline * Weighted kappa $(95 \% \mathrm{Cl})$ & $.396(.318,0.474)$ & $0.583(0.485,0.681)$ \\
\hline ** Weighted kappa $(95 \% \mathrm{CI})$ & $.248(.167,0.329)$ & $0.546(0.422,0.669)$ \\
\hline Diagnostic accuracy & BMI-MNA-SF & CC-MNA-SF \\
\hline Sensitivity & $34.2 \%$ & $75.8 \%$ \\
\hline Specificity & $100.0 \%$ & $83.9 \%$ \\
\hline PPV & $100.0 \%$ & $91.0 \%$ \\
\hline NPV & $41.5 \%$ & $61.8 \%$ \\
\hline Total Diagnostic accuracy & $55.12 \%$ & $78.41 \%$ \\
\hline *Malnutrition, risk of malnutrition, well-nourished & \\
\hline **"Malnutrition and risk of malnutrition", well-nourished & \\
\hline
\end{tabular}

The area under ROC curves using the MNA long-form as golden standard the area showed the highest values 0.908 for BM-MNA-SF and 0.880 for CC-MNA-SF (Fig. 2). AUC $(95 \% \mathrm{Cl})$ value indicates that both version MNA-SF had excellent diagnostic accuracy to diagnosis malnutrition with overall accuracy of $90.8 \%(86.5,95.1)$ for BMI-MNA-SF and for CC-MNA-SF 88.0\% $(83.1,92.9)$. In addition, Maximum Youden's index calculated using ROC curve was 0.654 and 0.597 for BMI-MNA-SF and CC-MNA-SF respectively. At these Youden's index values, the newly developed optimal cut off value for BMI-MNA-SF tool was 12.5 , and 10.5 for CC-MNA-SF to detect the markers of malnutrition (i.e. merged at risk of malnutrition and malnutrition). Based on new cut off value, BMI-MNA-SF total score $<12.5$ points as markers of malnutrition, sensitivity increased to $82.1 \%$, and specificity decreased to $83.3 \%$ while for CCMNA-SF total score $<10.5$ points as markers of malnutrition, sensitivity increased to $83.9 \%$, and specificity decreased to $75.8 \%$

\section{Discussion}

In this study, the BMI-MNA-SF and CC-MNA-SF had acceptable internal consistency within its six items of Cronbach's alpha value 0.205 and 0.319 respectively. In addition, BMI-MNA-SF and CC-MNA-SF showed significant criterion related validity with MNA long-form of Spearman's rho $\left(r_{s}\right) 0.771$ and 0.759 
respectively. Moreover, BMI-MNA-SF and CC-MNA-SF had a moderate agreement with MNA long-form of weighted kappa 0.396 and 0.583 respectively.

According to the originally established cut off value, both version MNA-SFs showed a strong overall diagnostic accuracy. Results for CC-MNA-SF slightly lower than the BMI-MNA-SF this might explained by characteristics of CC-score. Moreover, both version shows high PPV than NPV this might due to malnutrition prevalence. This study showed higher specificity higher as compared to study done in Germany(39) and Spain(40) for both version. Whereas, showed lower than the original developers of sensitivity and specificity (25). The variation in sensitivity and specificity may due to the setting and selection of the golden standard to validate the MNA.

However, according to newly developed best fit cut-off value for BMI-MNA-SF score of $<12.5$ (at Youden's index maximum 0.654 ) to detect markers malnutrition sensitivity increased to $82.1 \%$, and specificity decreased to $83.3 \%$ while for CC-MNA-SF score $<10.5$ (at Youden's index maximum 0.597 ) increased to $83.9 \%$, and specificity decreased to $75.8 \%$. In this study, BMI-MNA-SF correctly classify $65.4 \%$ while CCMNA-SF correctly classify $59.7 \%$ malnutrition. Moreover, the new cut off value brings sensitivity than the original cut off points. Therefore, further studies needed to evaluate newly developed cut-off value for Ethiopian elders using dietary and biomarkers as golden standard.

Lastly, this study had its limitation. One of this study limitation was combined different nutritional assessment methods were not used. Especially, the dietary assessment and biochemical were not assessed. Other limitation in this study only one golden standard used to validate.

\section{Conclusion}

This study showed that both versions of MNA short-form was valid and reliable tools for Ethiopian elders. Nevertheless, this study did not shows cost effectiveness due to the nature of cross-sectional study. Therefore, better to do future study that assess cost effectiveness of MNA short-form.

\section{Abbreviations}

AUC $=$ Area Under Curve

$\mathrm{BMI}=$ Body Mass Index

$\mathrm{CC}=$ Calf Circumference

$\mathrm{Cl}=$ Confidence Interval

MNA = Mini Nutritional Assessment

MNA-LF = Mini Nutritional Assessment Long-Form 
MNA-SF= Mini Nutritional Assessment Short-Form

MUAC = Mid-Upper Arm Circumference

NPV=Negative Predictive Value

PPV $=$ Positive Predictive Value

ROC-Curve $=$ Receiver-Operating Characteristic Curve

\section{Declarations}

\section{Ethical standards Disclosure}

This study was conducted according to the guidelines laid down in the World Medical Association (WMA) Declaration of Helsinki and all procedures involving research study participants were reviewed and approved by Jimma University, Institute of Health, Ethical review committee (ERC). Written informed consent was obtained from all participants.

\section{Consents for publication}

Not applicable

\section{Availability of data and materials}

Not applicable

\section{Competing of interest}

There is no competing interests.

\section{Funding}

This study was fully funded by Jimma University, Institute of Health, and Directorate of post-graduate studies. This organization had no any role in data collection, analysis, interpretation data and manuscript writing. All done by author.

\section{Author's contribution}

This manuscript done only one author. The corresponding author done all. 


\section{Acknowledgements}

I would like to Institute of health, faculty of Public Health, Jimma University. I also grateful to the study participants, data collectors and supervisors.

\section{References}

1. Ethiopia Ministry of labor and social affairs. National plan of action on older persons from 1998 to 2007 E.C. 2013.

2. United Nations. Department of Economic and Social affairs population division:World population ageing. ST/ESA/SEERRAA/430. 2019.

3. WHO. Available from: https://www.who.int/health-topics/ageing\#tab=tab_1

4. Ethiopia FDR population census commission. Summar and statistical report of 2007 population and housing census :population size by age and sex. Central statistical agency(CSA) of Ethiopia,Addis Ababa. 2014.

5. Worldometers. Population of Ethiopia (2019 and historical) 2019. Available from: https://www.worldometers.info/world-population/ethiopia-population.

6. Hayflick L. Biological aging is no longer an solved problem. Ann NY Acad Sci 2007.

7. Brownie S. Why are elderly individuals at risk of nutritional deficiency. International Journal of Nursing Practice 2006.

8. Help age Intrnational. Vulnerability of older people in Ethiopia: The case of Oromia ,Amahra and SNNP regional states Help age Intrnational ,Addis Ababa 2013.

9. Malnourished :key facts [Internet]. 2018. Available from: available at : http's//www.who.int/newsroom/fact-sheets/detail/malnutrition.

10. Cederholm T BI, Barazzoni R, Bauer J,Van Gossum A,Klek S, et al. . Diagnostic criteria for malnutrition- an ESPEN consensus statement. clin nutr 2015.

11. Skates $\mathrm{JJ}$, Anthony PS,. identifying geriatric malnutrition in nursing practice :the mini nutritional assessment(MNA) dan evidence-based screening tool J Gerontol Nurs 2012.

12. Morley JE. Anorexia of aging :Physiologic and pathologic. AM J Clin Nutr. 1997.

13. K.E.Charlton AK, Johanna H.,.. The MNA,but not DETERMINE,screening tool is a valid indicator of nutritional status in elderly Africans Nutrition. 2007. 
14. Sara A. Nutritional status and helath related qaulity of life among elderly in rural area in Egypt EJGG. 2018.

15. Haile mariam, Pragya S,Tigist F. Evalution of mini nutrition assessment (MNA) tool among community dwelling elderly in urban community of Hawassa city,Southern Ethiopia. BMC Nutrition. 2016.

16. Eglseer D, Halfens R,Lohrmann C,. Is the presence of a validated malnutrition screening tool associated with better nutritional care in hospitaled patients Nutrition. 2017.

17. Skipper A, Ferguson M,Thompson K,Castellanos VH,Porcari J. Nutrition screening tools : an analysis of the evidence. JPEN J Parenter Enteral Nutr. 2012.

18. Kondrup, Allison P,Elia M, Vellas B, Plauth M. ESPEN Guide line for nutrition screening 2002 Clinical nutrition 2003.

19. Skates JJ, Anthony PS. identifying geriatric malnutrition in nursing practice :the mini nutritional assessment(MNA) dan evidence-based screening tool J Gerontol Nurs. 2012.

20. Jones JM. Validity of nutritional screening and assessment tools. Nutrition 2004.

21. Marion S, Maria E ,Villars H, Gabor Ab and Vellas B. The Mini Nutritional Assessment (MNA) after 20 years of research and clinical practice. Reviews in Clinical Gerontology 2007.

22. Guigoz Y. Mini nutritional assessment: A practice assessment tool for grading the nutritional state of elderly patients. Facts and research in gerontology supplement 1994

23. Guigoz $\mathrm{Y}$. The mini nutritional assessment (MNA) review of the literature : what doest it tells? J Nutr health and ageing 2006.

24. Power L, Mullally D,Gibney E,Clarke $M$, Visser $M$, Dorothee $V$ et al. A reveiw of the validity of mal nutrition screening tools used in older adults in community and health care setting clinical nutrition. 2018.

25. Rubenstein L. Screening for under nutrition in geriatric practice : developing the short form mini nutritional assessment (MNA SF). Journals of gerontology series A : Biological sciences and medical sciences 2001

26. Elia $\mathrm{M}$ et al. Aguide to the malnutrition malnutrition universal screening tool (MUST) for adult 2003.

27. Kruizenga et al. Development and validation of a hospital screening tool for malnutrition : the short form nutritional assessment queostinairre (SNAQ). CLinical nutrition 2005.

28. Charlton et al. Development of a novel nutrition screening tool for use in elderly South Africans. Public heath nutrition 2005. 
29. Kaiser MJ, Bauer JM,Anthony P,Guigoz Y,Vellas B,Tsai AC et al. Validation of the mini nutritional assessment short form (MNA-SF) : A practical tool for identification of nutritional status $\mathrm{J}$ Nutr health and ageing 2009.

30. Nazan S, Buket K. Evaluation of nutritional status of elderly patients presenting to the family health center. 2018.

31. Mesfin Agachew Woldekidan, Demewoz Haile, Bilal Shikur \&,Seifu Hagos, Gebreyesus. Validity of Mini Nutritional Assessment tool among an elderly population

in Yeka sub-city, Addis Ababa, Ethiopia. South African Journal of Clinical Nutrition. 2020; .

32. Mini nutritional assessment [Internet]. Nestle nutrition institute. 2009. Available from: www.mnaelderly.com.

33. Guide to complete mini nutritional assessment [Internet]. Nestle nutrition institute. 2010. Available from: www.mna-elderly.com.

34. Garson G. Validity and reliability Statistical associations : avaliable at: wwwstatisticalassociatecom wwwstatisticalassociatecom 2016.

35. Mukaka M. Statitics corner : A guide appropriate use of correlation coefficient in medical research. Malawi medical journal. 2012.

36. Landis G, Koch J. The measurement of observer agreement for categorical data. Biometric. 1977.

37. Zhu W, Zeng N,Wang N. Sensitivity ,specificity,accuracy,assocaited confidenfce interval and ROC analysis with practical SAS implementation NESUG: health cares and life science. 2010.

38. Youden WJ. Index for rating diagnostic tests National bureau of standard Washington DC: Cancr ,doi:101002/1097-0142 1950.

39. Kaiser MJ, Bauer,Uter W,Donini ML,Volkert D,Diekmann $\mathrm{R}$ et al. Prospective validation of the modified mini nutritional assessment short form in the community,nursing home and rehabilitation setting. American geriatric society. 2011.

40. Garcia et al. Validation of the revised mini nutritional assessment short form in nursing homes in Spain Journal health and ageing 2013.

\section{Figures}




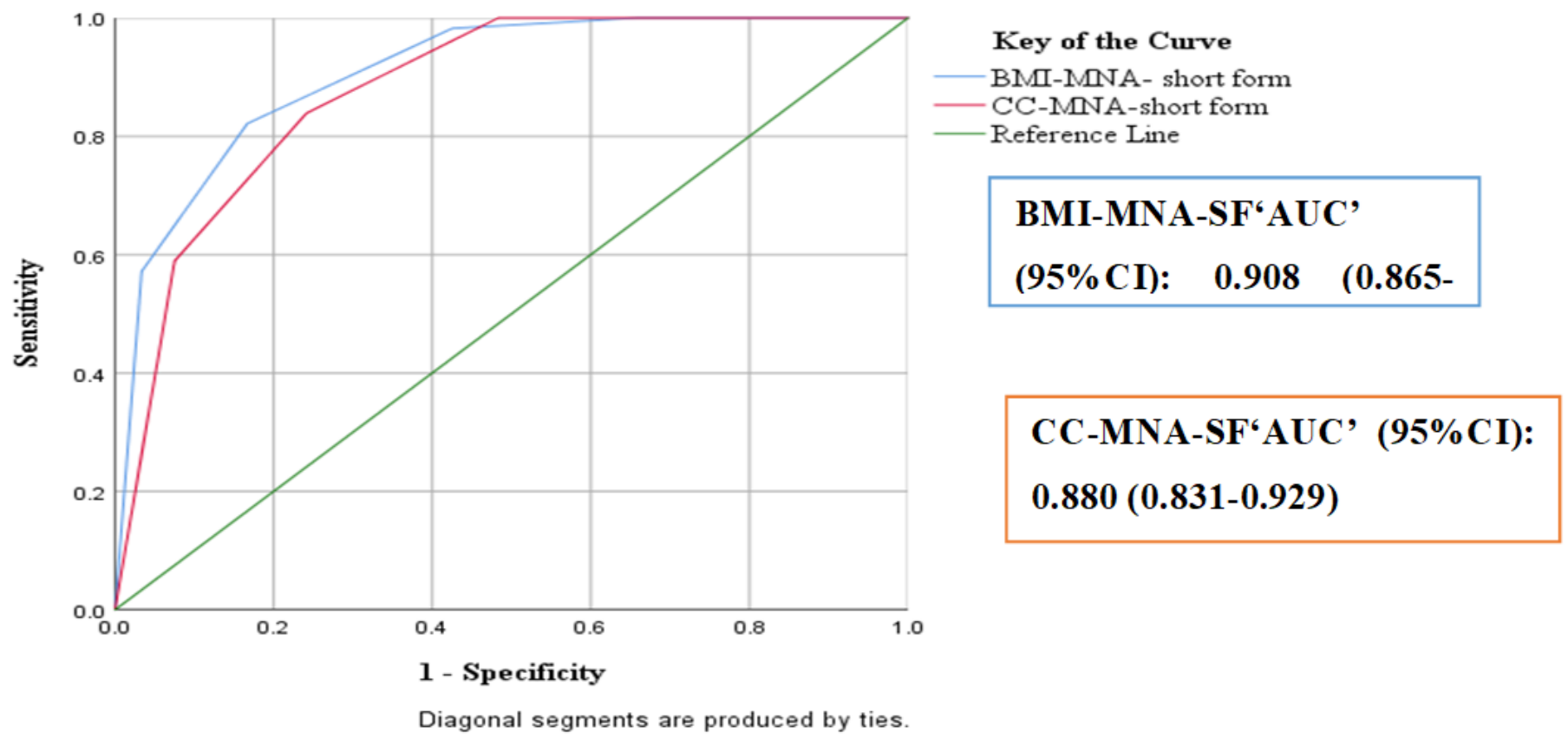

Figure 1

The ROC curves of one hundred and seventy-six samples for the MNA-short form tools as compared to MNA-long form of participant elderly people aged 60 and above years in the community, Meki town, East Ethiopia, 2020. 